\title{
Effects of TESTIN Gene Expression on Proliferation and Migration of the 5-8F Nasopharyngeal Carcinoma Cell Line
}

\author{
Zhun Zhong ${ }^{1,2}$, Fei Zhang ${ }^{3}$, Shu-Cheng Yin ${ }^{1 *}$
}

\begin{abstract}
Purpose: To investigate effects of the TESTIN (TES) gene on proliferation and migration of highly metastatic nasopharyngeal carcinoma cell line 5-8F and the related mechanisms. Materials and Methods: The target gene of human nasopharyngeal carcinoma cell line 5-8F was amplified by PCR and cloned into the empty plasmid pEGFP-N1 to construct a eukaryotic expression vector pEGFP-N1-TES. This was then transfected into 5-8F cells. MTT assays, flow cytometry and scratch wound tests were used to detect the proliferation and migration of transfected 5-8F cells. Results: A cell model with stable and high expression of TES gene was successfully established. MTT assays showed that the OD value of 5-8F/TES cells was markedly lower than that of 5-8F/GFP cells and 5-8F cells $(p<0.05)$. Flow cytometry showed that the apoptosis rate of 5-8F/TES cells was prominently increased compared with $5-8 \mathrm{~F} / \mathrm{GFP}$ cells and $5-8 \mathrm{~F}$ cells $(p<0.05)$. In vitro scratch wound assays showed that, the width of the wound area of 5-8F/TES cells narrowed slightly, while the width of the wound area of 5-8F/ GFP cells and 5-8F cells narrowed sharply, suggesting that the TES overexpression could inhibit the migration ability. Conclusions: TES gene expression remarkably inhibits the proliferation of human nasopharyngeal carcinoma cell line $\mathbf{5 - 8 \mathrm { F }}$ and reduces its migration in vitro. Thus, it may be a potential tumor suppressor gene for nasopharyngeal carcinoma.
\end{abstract}

Keywords: TESTIN gene - nasopharyngeal carcinoma - proliferation - migration

Asian Pac J Cancer Prev, 16 (6), 2555-2559

\section{Introduction}

Nasopharyngeal carcinoma is one of the common clinical head and neck cancers with a relatively young age of onset. It predominantly occurs in southern China, Southeast Asia, North Africa and Alaska, with an incidence rate of about 30-50 per 100 thousands (Wei and Sham, 2005; Sun et al., 2006). Although the clinical studies on nasopharyngeal carcinoma have made great progress, the overall 5-year survival rate of nasopharyngeal carcinoma remains low, indicating that the clinical studies are still insufficient for improving the survival rate of nasopharyngeal carcinoma. The etiological pathogenesis of nasopharyngeal carcinoma should be studied in-depth, especially on the nasopharyngeal carcinoma-related genes. In recent years, $7 q 31$ is found to be an area with a high frequency of loss of heterozygosity in a variety of malignant tumors ( Latil et al., 1995; Zenklusen et al., 1995; Bieche et al., 1997; Edelson et al., 1997; Nishizuka et al., 1997; Matsuura et al., 1998), suggesting that there may be tumor suppressor genes in this region. TES gene locates on FRA7G fragile site of 7q31.2, encoding a protein sequence comprising 421 amino acid residues. TES is widely expressed in normal tissues but deficit in a variety of primary tumors and tumor cell lines, especially in the cell lines of gastric carcinoma, breast cancer, colorectal cancer, glioma, ovarian cancer, leukemia and endometrial carcinoma, suggesting that it may be a candidate tumor suppressor gene (Zenklusen et al., 1995; Bieche et al., 1997; Edelson et al., 1997; Nishizuka et al., 1997; Weeks et al., 2010; Gu et al., 2014). This study was conducted to investigate the effects of TES gene overexpression on the proliferation and migration of human nasopharyngeal carcinoma cell 5-8F, and explore the related mechanism.

\section{Materials and Methods}

\section{Cell culture}

Human nasopharyngeal carcinoma 5-8F cells (subtype of SUNE-1 cell, with high metastasis and high tumorigenicity; Shanghai Cell Bank of Chinese Academy of Sciences, Shanghai, China) were cultured in DMEM (Gibco® Life Technologies, NY, USA) supplemented with $10 \%$ fetal bovine serum (FBS, Gibco $®$ Life Technologies, NY, USA), $1 \%$ penicillin-streptomycin combination (Adamas Reagent, Ltd., Shanghai, China) and 4.5\% Glucose (Adamas Reagent, Ltd., Shanghai, China), in a circumstance of $5 \% \mathrm{CO}_{2}$ and $37^{\circ} \mathrm{C}$. The exponentially

${ }^{1}$ Department of Otolaryngology Head and Neck Surgery, Zhongnan Hospital of Wuhan University, ${ }^{2}$ Department of Otolaryngology, Zhongshan Hospital of Hubei Province, ${ }^{3}$ Department of Otorhinolaryngology, Maternal and Child Health Hospital of Hubei Province, Wuhan, Hubei, China*For correspondence: ShuchengYincn@163.com 
growing cells were used for the subsequent experiments.

Construction and identification of TES recombinant plasmid

Based on the cDNA library, the specific primer pairs for TES gene were designed and synthesized by Sangon Biotech (Shanghai) Co., Ltd. (Shanghai, China), according to the digestion patterns of TES gene and vector pEGFP-N1. The forward and reverse primers contained a specific restriction site, respectively. TES gene fragment amplified from human nasopharyngeal carcinoma tissue DNA was inserted into eukaryotic expression vector pEGFP-N1 in vitro to construct a recombinant plasmid pEGFP-N1-TES. The recombinant plasmid pEGFP-N1TES was identified by digestion and sequencing before it was transfected into 5-8F cells.

Construction and identification of stably transfected cell line

The recombinant plasmid pEGFP-N1-TES was transfected into $5-8 \mathrm{~F}$ cells using liposome transfection method. At the $48 \mathrm{~h}$ after the transfection, cells were treated with G418 (500 mg/L) screening medium (Gibco ${ }^{\circledR}$ Life Technologies, NY, USA) for 3 weeks. Then the resistant clones were picked out to culture extensively. A 5-8F/TES cell line stably expressing TES gene was established. A parallel transfection with empty vector pEGFP-N1 to 5-8F cells was also carried out as a control group $(5-8 \mathrm{~F} / \mathrm{GFP})$. The mRNA and protein expressions of TES gene were identified using conventional RT-PCR and Western-blot methods, respectively.

\section{MTT assay}

$5-8 \mathrm{~F}, 5-8 \mathrm{~F} / \mathrm{TES}$ and $5-8 \mathrm{~F} / \mathrm{GFP}$ cells were seeded in 96-well plates at a density of $1 \times 10^{4}$ cell/well, respectively. Then the cells were incubated in a circumstance of $5 \% \mathrm{CO}_{2}$ and $37^{\circ} \mathrm{C}$ for $72 \mathrm{~h}$. As the cells grew to $80 \%$ of confluence, the freshly prepared MTT solution $(5 \mathrm{mg} / \mathrm{ml})$ was added to each well ( $50 \mu \mathrm{L} /$ well $)$, and then incubated for additional $2 \mathrm{~h}$. Subsequently, the supernatant was removed from the well and $200 \mu \mathrm{L}$ DMSO was added to the well. After shaking, the absorbance of each well at $450 \mathrm{~nm}$ was measured using a microplate reader. The growth curves of the $5-8 \mathrm{~F}$ cells treated with different factors were plotted.

\section{Flow cytometry}

Cells in active phase were seeded in 6-well plate $\left(1 \times 10^{4}\right.$ cell/well $)$ and incubated in $3 \mathrm{~mL} 1640$ medium supplemented with $10 \% \mathrm{FBS}$ at $37^{\circ} \mathrm{C}$ and in $5 \% \mathrm{CO}_{2}$. As the cells grew to the logarithmic phase, appropriate drugs were added to the wells. After that, the culture supernatant of each well was collected into BD Falcon tubes (Becton, Dickinson and Company, NY, USA). The cells in the 6 -well plates were also collected by digestion with $1 \mathrm{~mL}$ $0.25 \%$ trypsin. After washed by pre-cooled PBS twice, the cells were re-suspended in $300 \mu \mathrm{L}$ binding buffer and mixed with $5 \mu \mathrm{L}$ Annexin V-PE/7-AAD and $5 \mu \mathrm{L}$ propidium iodide successively, followed by incubation at room temperature in the dark for 5-15 min. The apoptosis rate of the cells was detected on a flow cytometer within $1 \mathrm{~h}$.
Scratch wound assay

The cells were plated in a 6 -well plate $\left(4 \times 10^{5}\right.$ cell/ well), and maintained in $2 \mathrm{~mL} 1640$ medium the day before transfection. At the $48 \mathrm{~h}$ after transfection, 5 parallel scratches were made with a P200 micropipette tip (Axygen Scientific, Inc., CA, USA) in each well with intervals of $0.5-1 \mathrm{~cm}$. At the next day, the plate was washed with PBS twice to remove the detached and floating cells and cellular debris and continued to incubated with $2 \mathrm{~mL}$ serum-free $\mathrm{DMEM}$ in $5 \% \mathrm{CO}_{2}$ and at $37^{\circ} \mathrm{C}$ for $5 \mathrm{~h}$. Then the medium was replaced by complete 1640 medium and incubated in $5 \% \mathrm{CO}_{2}$ again. Photographs of cells invading the scratch were taken at $0,12,24$ and $48 \mathrm{~h}$, respectively, for assessing the effect of TES overexpression on cell migration.

\section{Statistical analysis}

This study was designed using complete randomized design method. All statistical analysis was carried out using SPSS 17.0 software (SPSS Inc., Chicago, IL, USA). Data are presented as mean \pm SD. Comparisons between two groups were performed using $t$ test and comparisons between multiple groups were performed using onefactor analysis of variance. $\mathrm{P}<0.05$ was considered as statistically significant.

\section{Results}

Construction and identification of TES recombinant plasmid

The recombinant plasmid pEGFP-N1-TES was identified by PCR and DNA sequencing. The PCR products (size: $1284 \mathrm{bp}$ ) analyzed by agarose gel electrophoresis showed a bright band between the marker $1000 \mathrm{bp}$ and $2000 \mathrm{bp}$ (Figure 1). The DNA sequencing of PCR products showed the sequence of the clonal gene was consistent to TES gene sequence in the GeneBank (Figure 2).

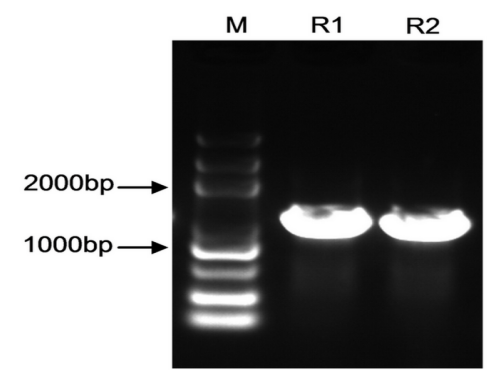

Figure 1. PCR Products of two Recombinant Plasmid Bacterial Colonies Analyzed by Gel Electrophoresis (M, D2000 Plus Marker; R1 and R2, two Bacterial Colonies Transformed with Recombinant Plasmid pEGFP-N1-TES)

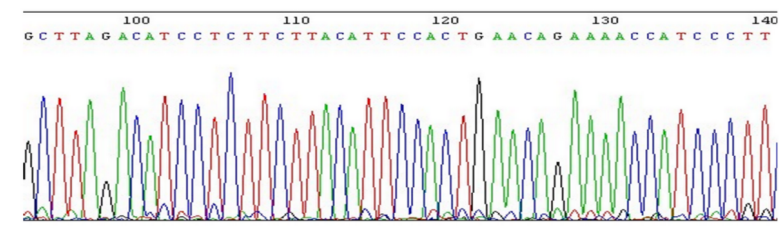

Figure 2.DNA Sequencing of the Recombinant Plasmid pEGFP-N1-TES 
Construction, screening and identification of cells transfected with TES gene

The relative expression of TES mRNA in the selected clones $(5-8 \mathrm{~F} / \mathrm{TES})$ was significantly higher than that in the $5-8 \mathrm{~F} / \mathrm{GFP}$ cells and 5-8F cells $(p<0.05)$ (Figure 3 ). This indicated that the TES-overexpression cell line 5-8F/TES was established successfully. TES protein expression in the selected clones was also remarkably higher than that in the 5-8F/GFP cells and 5-8F cells $(p<0.01)$ (Figure 4).

\section{Effect of TES overexpression on growth of 5-8F cells}

The results of MTT assay showed that, the OD value of $5-8 \mathrm{~F} / \mathrm{TES}$ cells was markedly lower than that of $5-8 \mathrm{~F} /$ GFP cells and 5-8F cells and the differences tended to be more significant in a time-dependant manner $(p<0.05)$, while the difference between OD values of 5-8F/GFP cells and 5-8F cells was not significant $(p>0.05)$. This indicated

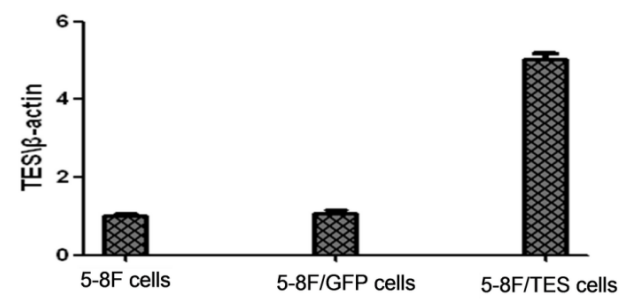

Figure 3. mRNA Expression of TES Gene in the Selected Clones (5-8F/TES), 5-8F/GFP Cells and 5-8F Cells Determined by RT-PCR
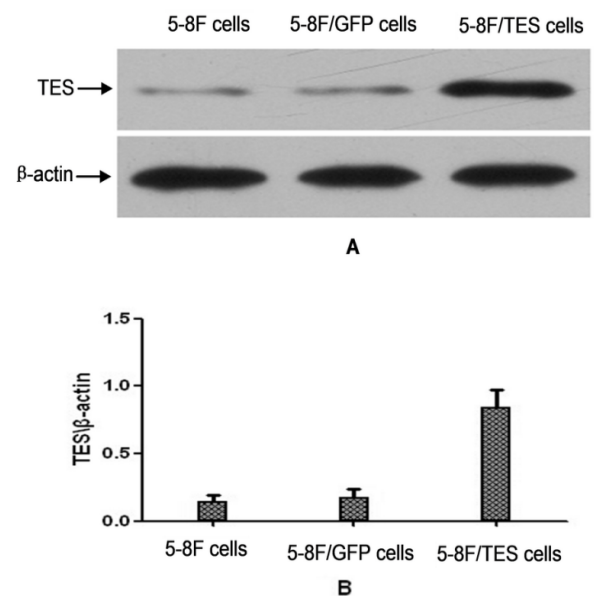

Figure 4. Protein Expression of TES in the Selected Clones (5-8F/TES), 5-8F/GFP Cells and 5-8F Cells Determined by Western-blot. The protein expressions of TES in 5-8F cells, 5-8F/GFP cells and 5-8F/TES cells were 0.14 , 0.17 and 0.84 , respectively, relative to gray value of $\beta$-actin

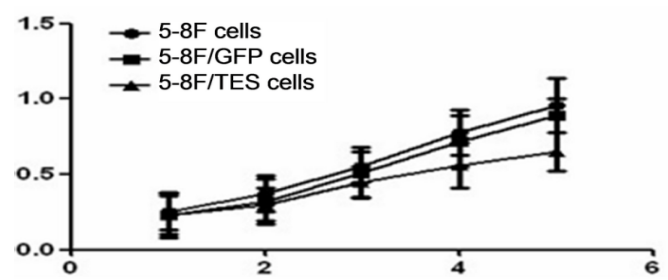

Figure 5. Growth Curves of 5-8F Cells, 5-8F/GFP Cells and 5-8F/TES Cells Determined by MTT Assay
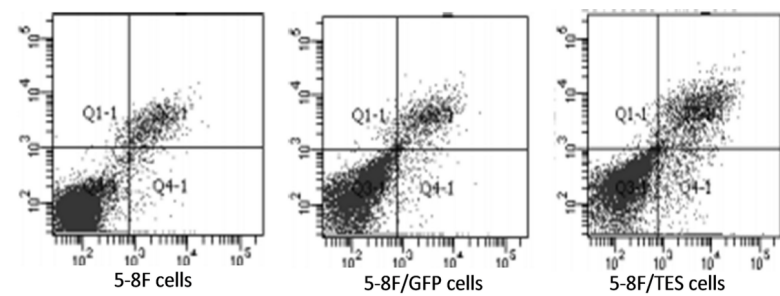

Figure 6. Flow Cytometry Analysis of the Apoptosis Rates of 5-8F Cells, 5-8F/GFP Cells and 5-8F/TES Cells

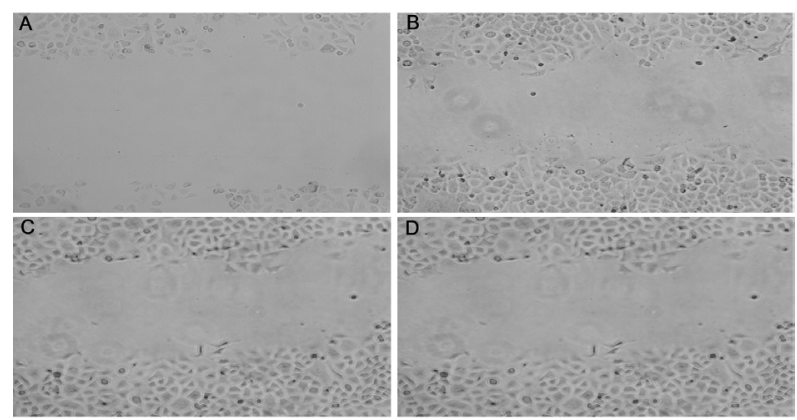

Figure 7. Scratch Wound of Cells (100x). (A) Baseline, 0 h. (B) 5-8F/TES cells, 24 h. (C) 5-8F/GFP cells, 24 h. (D) $5-8 \mathrm{~F}$ cells, $24 \mathrm{~h}$

that, TES could effectively inhibit the proliferation of 5-8F cells (Figure 5).

Effect of TES overexpression on apoptosis of 5-8F cells

The flow cytometry results showed that, the apoptosis rate of $5-8 \mathrm{~F} / \mathrm{TES}$ cells was prominently increased compared with $5-8 \mathrm{~F} / \mathrm{GFP}$ cells and $5-8 \mathrm{~F}$ cells $(p<0.05)$ (Figure 6). However, the apoptosis rate of 5-8F/GFP cells showed no significantly difference from that of $5-8 \mathrm{~F}$ cells $(p>0.05)$. This indicated that, the TES overexpression could increase the apoptosis of 5-8F cells.

Effect of TES expression on migration ability of 5-8F cells

The effect of TES expression on migration ability of $5-8 \mathrm{~F}$ cells was examined by in vitro scratch wound assay. As observed under a phase contrast microscope, the width of the wound area of 5-8F/TES cells narrowed slightly, while the width of the wound area of 5-8F/GFP cells and 5-8F cells narrowed sharply, suggesting that the TES overexpression could inhibit the migration ability of 5-8F cells (Figure 7).

\section{Discussion}

The overall 5-year survival of nasopharyngeal carcinoma is only about $40 \%$, while the prognostic 5-year survival of nasopharyngeal carcinoma at early stage is up to $80 \%$ (Ma et al., 2001; Lin et al., 2003). Therefore, early diagnosis and treatment of nasopharyngeal carcinoma can be of a great importance. Currently, the etiology of nasopharyngeal carcinoma is not clear yet. Infection with EB virus, environment, diet and genetic factors may be important incentives in the occurrence of nasopharyngeal carcinoma (Tobias et al., 2001; Bingle and Craven, 2002; Bingle and Craven, 2004; Bingle and Gorr, 2004; Zhou et al., 2006).

TES gene is an important tumor suppressor gene found 
recently, locating at the fragile site FRA7G of human chromosome $7 \mathrm{q} 31.1 / 2$ and encoding a protein containing 421 amino acid residues, which exerts tumor suppressor in epithelial originated tumors. It is worthy to be pointed out that, TES gene highly expressed in mouse testis is completely different with human TES gene, but they are garbled in previous study (Mruk and Cheng, 2012). There are several cysteine-rich LIM structural domains in the C-terminal of TES gene, such as lin-11, isl-1 and mec3 domains (Way and Chalfie, 1988; Freyd et al., 1990; Karsson et al., 1990). Studies have confirmed that, LIM structural domains can serve as an interface for protein identification to provide a variety of joints and supports for protein interactions, so as to mediate the interaction between proteins (Arber and Caroni, 1996; Adrmas and Beckerle, 2004; Magno et al., 2011). To date, several lines of evidence indicate that, TES may be a candidate tumor suppressor gene. Tobias et al. (2001) find that, the deficit of TES is associated with hypermethylation of $\mathrm{CpG}$ islands in the $\mathrm{COOH}$ terminus and the overexpression of TES can significantly inhibit the growth of ZR-75 cells, T47D cells, HeLa cells and OVCAR5 cells. In addition, Drusco et al. (2005) have established a TES KO mice model and find that, the incidence rate of carcinogen NMBA-induced gastric cancer in TES KO mice is remarkably higher than that in the TES wild-type mice, which confirms the tumor suppressor function of TES gene in vivo. Zhu et al. (2012) have successfully constructed a stable expression cell line with TES recombinant plasmid in breast cancer cells and implanted it in nude mice. Results show that, the invasiveness and tumorigenicity of the tumor cells transfected with TES recombinant plasmid are strikingly decreased. Manuela et al. (2013) also find that, TES gene can be used as an independent molecular indicator for the prognosis of breast cancer. However, how TES gene plays its roles in the development and progression of tumors is not clear yet, especially for its mechanism of action in the invasion and metastasis of tumors. To date, there are few studies on the effects of TES on the head and neck tumors, especially on nasopharyngeal carcinoma. In this study, the TES gene is isolated from human nasopharyngeal carcinoma cells and inserted into PEGFP-N1 vector to construct a recombinant plasmid PEGFP-N1-TES in vitro. As confirmed by PCR and agarose gel electrophoresis, the PCR product size is $1284 \mathrm{bp}$, consistent with the predicted size. DNA sequencing of the PCR product also confirms that, the target sequence is consistent with that reported in the literatures, suggesting that the TES recombinant plasmid is successfully constructed. Then the TES recombinant plasmid is transfected into human nasopharyngeal carcinoma 5-8F cells and identified by RT-PCR and western-blot method. The results show that, the mRNA and protein expression of TES gene in 5-8F cells transfected with PEGFP-N1-TES are significantly higher than that in 5-8F cells transfected with empty vector and untransfected cells. The $5-8 \mathrm{~F}$ cells transfected with exogenous TES gene highly express TES, indicating that the cell model stably transfected with TES gene is successfully established.

MTT assay is a method used to evaluate cell proliferation, which can well reflect the growth characteristics of tumor cells in vivo. In this study, the results of MTT assay show that, the proliferation of 5-8F cells transfected with TES gene is significantly reduced compared with that of the cells transfected with empty vector and untransfected cells, suggesting that TES can effectively inhibit the proliferation of 5-8F cells. Flow cytometry is one testing means used for quantitative analysis and sorting of single cell or other biological particles at the function level. It has become the most advanced cellular quantitative analysis technique. In this study, the results of flow cytometry show that, TES can inhibit the in vitro growth of 5-8F cells by influencing the apoptosis and then inhibit their proliferation. Currently, TES gene has been confirmed to inhibit the tumor proliferation by increasing apoptosis in several tumor cell lines, but its pro-apoptotic mechanism is not clear yet. Thus, more studies should be carried out on it in future. Scratch wound assay is often used to evaluate the motility and migration of cells by calculating the cell number invading the scratch zone at different time points. In this study, it is used as an important indicator to evaluate the invasiveness and metastasis of cells. Results show that, the TES overexpression could reduce the motility and migration of $5-8 \mathrm{~F}$ cells.

In conclusion, TES gene can inhibit the growth and migration of nasopharyngeal carcinoma cells. In future, we will further study the association of TES gene with the invasiveness and metastasis of nasopharyngeal carcinoma by cell invasion assay and animal experiments. We hoped to provide more important clues for the exploration of nasopharyngeal carcinoma development and the individualized gene therapy for nasopharyngeal carcinoma patients.

\section{Acknowledgements}

This work was supported by grant from the Natural Science Foundation of Hubei Province, China (2009CBD420).

\section{References}

Adrmas JL, Beckerle MC (2004). The LIM domain: from the cytoskeleton to the nucleus. Nat Rev Mol Cell Biol, 5, 920-31.

Arber S, Caroni P (1996). Specificity of single LIM motifs in targeting and LIM/LIM interactions in situ. Genes Dev, 10, 289-300.

Bieche I, Khodja A, Driouch K, et al (1997). Genetic alteration mapping on chromosome 7 in primary breast cancer. Clin Cancer Res, 3, 1009-16.

Bingle CD, Craven CJ (2002). PLUNC: a novel family of candidate host defence proteins expressed in the upper airways and nasopharynx. Hum Mol Genet, 11, 937-43.

Bingle CD, Craven CJ (2004). Meet the relatives: a family of BPI- and LBP-related proteins. Trends Immunol, 25, 53-5.

Bingle CD, Gorr SU (2004). Host defense in oral and airway epithelia: chromosome 20 contributes a new protein family. Int J Biochem Cell Biol, 36, 2144-52.

Drusco A, Zanesi N, Roldo C, et al (2005). Knockout mice reveal a tumor suppressor function for Testin. Pnas, 102, 10947-51.

Edelson MI, Scherer SW, Tsui LC, et al (1997). Identification of a 1300 kilobase deletion unit on chromosome 7q31.3 
in invasive epithelial ovarian carcinomas. Oncogene, 14, 2979-84.

Freyd G Kim SK, Horvitz HR (1990). Novel cysteine-rich motif and homeo-domain in the product of the Caenorhabditis elegans cell lineage gem lin-11. Nature, 344, 876-9.

Gu Z, Ding K, Liang K, et al (2014). TESTIN suppresses tumor growth and invasion via manipulating cell cycle progression in endometrial carcinoma. Med Sci Monit, 20, 980-7.

Karsson O, thor S, Norberg T, et al (1990). Insulin gene enhancer binding protein Isl-1 is a member of a novel class of proteins containing both a homeo-and a Cys-His domain. Nature, 344, 879-82.

Latil A, Cussenol O, Fournier G, et al (1995). Loss of heterozygosity at $7 \mathrm{q} 31$ is a frequent and early event in prostate cancer. Clin Cancer Res, 1, 1385-9.

Lin JC, Jan JS, Hsu CY, et al (2003). Phase III study of concurrent chemmoradiotherapy versus radio-therapy alone for advanced nasopharyngeal caicinoma: positive effect on overall and progression-free survival. J Clin Oncol, 21, 631-7.

Ma J, Mai HQ, Hong MH, et al (2001). Results of a prospective randomized trial comparing neoadjuvant chemotherapy plus radiotherapy with radiotherapy alone in patients with locoregionally advanced nasopharyngeal carcinoma. J Clin Oncol, 19, 1350-7.

Magno AL, Ingley E, Brown SJ, et al (2011). Testin, a novel binding binding partner of the calciun-sensing receptormediated Rho-kinase signalling. Biochem Biophys Res Commun, 412, 584-9.

Manuela S, Sandra P, Costanzo L, et al (2013). Differential expression of testin and survivin in breast cancer subtypes. Oncology Rep, 30, 824-32.

Matsuura K, Shiga K, Yokoyama J, et al (1998). Loss of heterozygosity of chromosome $9 \mathrm{p} 21$ and $7 \mathrm{q} 31$ is correlated with high incidence of recurrent tumor in head and neck squamous cell carcinoma. Anticancer Res, 18, 453-8.

Mruk DD, Cheng CY (2012). Rat and mouse testicular testin is different from the human tumor suppressor gene TESTIN (Tes): Authors' response to the letter of Dr S. Kapoo. Spermatogenesis, $2,305$.

Nishizuka S, Tamura G, Terashima M, et al (1997). Commonly deleted region on the long arm of chromosome 7 in differentiated adenocarcinoma of the stomach. Br J Cancer, 76, 1567-71.

Sun Y, Yi H, Zhang PF, et al (2006). Identification of differential proteins in nasoparyngeal carcinoma cells with p53 silence by proteome analysis. Febs Lett, 581, 131-9.

Tobias ES, Hurlstone AF, MacKenzie E, et al (2001). The TES gene at $7 \mathrm{q} 31.1$ is methylated in tumours and encodes a novel growth-suppressing LIM domain protein. Oncogene, 20, $2844-53$.

Way JC, Chalfie M (1988). Mec-3, a homeobox-containing gene that specifies differentiation of the touch receptor neurons in C elegans. Cell, 54, 5-16.

Weeks RJ, Kees UR, Song S, et al (2010). Silencing of TESTIN by dense biallelic promoter methylation is the most commom molecular event in childhood acute lymphoblastic leukaemia. Mol Cancer, 9, 163.

Wei WI, Sham JS (2005). Nasopharyngeal carcinoma. Lancet, 365, 2041-54.

Zenklusen JC, Thompson JC, Klein-Szanto AJ, et al (1995). Frequent loss of heterozygosity in human primary squamous cell and colon carcinomas at 7q31.1: evidence for a broad range tumor suppressor gene. Cancer Res, 55, 1347-50.

Zhou HD, Li GY, Yang YX, et al (2006). Intracellular colocalization of SPLUNCI protein with nanobacteria in nasopharyngeal carcinoma epithelia HNE1 cells depended on the bactericidal permeability increasing protein domain. Mol Immunol, 43, 1864-71.

Zhu J, Li X, Kong XN, et al (2012). Testin is a tumor suppressor and prognostic marker in breast cancer. Cancer Sci, 3, 2092-101. 